\title{
TECHNIQUE FOR FORMING AN RF SAMPLE SIGNAL FOR ACCELERATING FIELD FEEDBACK STABILIZATION IN TRAVELING WAVE LINACS
}

\author{
A. M. Vetter ${ }^{\#}$, Boeing Phantom Works, Seattle, WA
}

\begin{abstract}
Feedback loop techniques have been employed for decades in the stabilization of linac RF systems. Systems using these techniques require an input signal which represents the accelerating fields which are to be stabilized. Early implementations have taken a sample of the klystron output power as the source for this signal, thus enabling stabilization of fluctuations in the level and phase of klystron output due to variations in the high voltage pulse, droop in the driver amplifier gain, etc. However, fluctuations produced by beam loading, thermal drift of the linac section, and other effects which occur downstream from the sample point cannot be sensed or corrected using this approach.

A technique for combining samples of the input and output power from all of the traveling wave sections driven by a given klystron, enabling the system to respond to perturbations introduced at points throughout the RF system, has been developed. This approach is extended to both constant impedance and tapered section types. Described here are the laws which relate the phases and levels of the sample signals which are to be presented to the summing circuit to form the input to the stabilization loop.
\end{abstract}

\section{BACKGROUND}

The first visible FEL at the Boeing Physical Sciences Research Center was driven by an L band linac consisting of six traveling wave sections, each powered by a pulsed klystron stabilized by a feedback loop operating on a klystron output sample. The system worked well enough (after a settling period of 10-20 $\mu$ s) to stabilize the output power level to better than $1 \%$ and phase to better than $1^{\circ}$ for the duration of the $100-\mu \mathrm{s}$ macropulse. The perturbations corrected by the feedback loop were chiefly due to klystron high voltage ripple (principally due to residual PFN unflatness) and droop in the klystron driver amplifier output. With this simple scheme, operators succeeded in achieving lasing in the FEL, but found the accelerator drifted out of tune frequently, probably because of inadequate temperature stabilization of the linac sections.

Present plans for a second generation visible FEL based on pulsed linac technology call for increasing macropulse repetition rate from $2 \mathrm{~Hz}$ to $30 \mathrm{~Hz}$,

"Work supported by USA/SSDC under Contract DASG-60-97-C-0105.

${ }^{\text {\#} E m a i l: ~ a r t h u r . m . v e t t e r @ b o e i n g . c o m ~}$ macropulse length from 100 to $200 \mu \mathrm{s}$, and macropulse average beam current from $10 \mathrm{~mA}$ to $100 \mathrm{~mA}$. The first two items, constituting a 30 -fold RF duty factor increase to $0.6 \%$, will drive much greater thermal drifts in both the modulator output and the linac section cell tuning over minute time scales as PFN coils, pulse transformer, and linac sections heat up. The increased beam loading will cause a $\sim 5 \%$ linac energy droop (relative to low tune-up beam currents). These greatly enhanced perturbing effects could render the FEL virtually inoperable at its design output power unless the RF stabilization system is capable of stabilizing the accelerating voltage rather than solely the klystron output.

\section{APPROACH}

The $1.3 \mathrm{GHz}$ RF systems of the upgraded linac typically contain two traveling wave linac sections powered by a single klystron. Given that our traveling wave sections incorporate no provision for sampling internal RF fields, the best approximation to the total complex accelerating voltage $\boldsymbol{V}_{\text {system }}$ applied to the beam (by all the cells of both the sections of a given RF system) which can be determined by available measurements is an appropriately weighted average of phase-adjusted coupler cell accelerating voltages $\boldsymbol{V}_{b}, \boldsymbol{V}_{c}, \boldsymbol{V}_{e}$, and $\boldsymbol{V}_{f}$ (subscripts refer to points labeled in Fig. 1) which can be deduced from samples of the RF waves entering the input couplers and leaving the output couplers. That is,

$$
\boldsymbol{V}_{\text {ssstem }}=D_{b} \boldsymbol{V}_{b}+e^{i \Phi_{b c}} D_{c} \boldsymbol{V}_{c}+e^{i \Phi_{b e}}\left(D_{e} \boldsymbol{V}_{e}+e^{i \Phi_{e f}} D_{f} \boldsymbol{V}_{f}\right),
$$

where the $D$ 's are the weighting coefficients (determined as described below) and $\Phi_{x y}$ represents the phase elapsed during the time required for beam electrons to move from point $x$ to point $y$. This average reflects the effects of beam loading along both real and imaginary axes, thermal drift in phase length of the sections, etc.

As noted above, the coupler cell voltages are determined from the sample signal voltages $\boldsymbol{V}_{1} \ldots \boldsymbol{V}_{4}$ which are added in the power combiner. These sample signals must be correctly related in phase and signal level if their sum is to be proportional to $\boldsymbol{V}_{\text {ssstem }}$. The remainder of this paper describes how the weighting coefficients are determined and gives the relative phase and level relationships which must be obtained between sample signals for our upgraded FEL linac. 


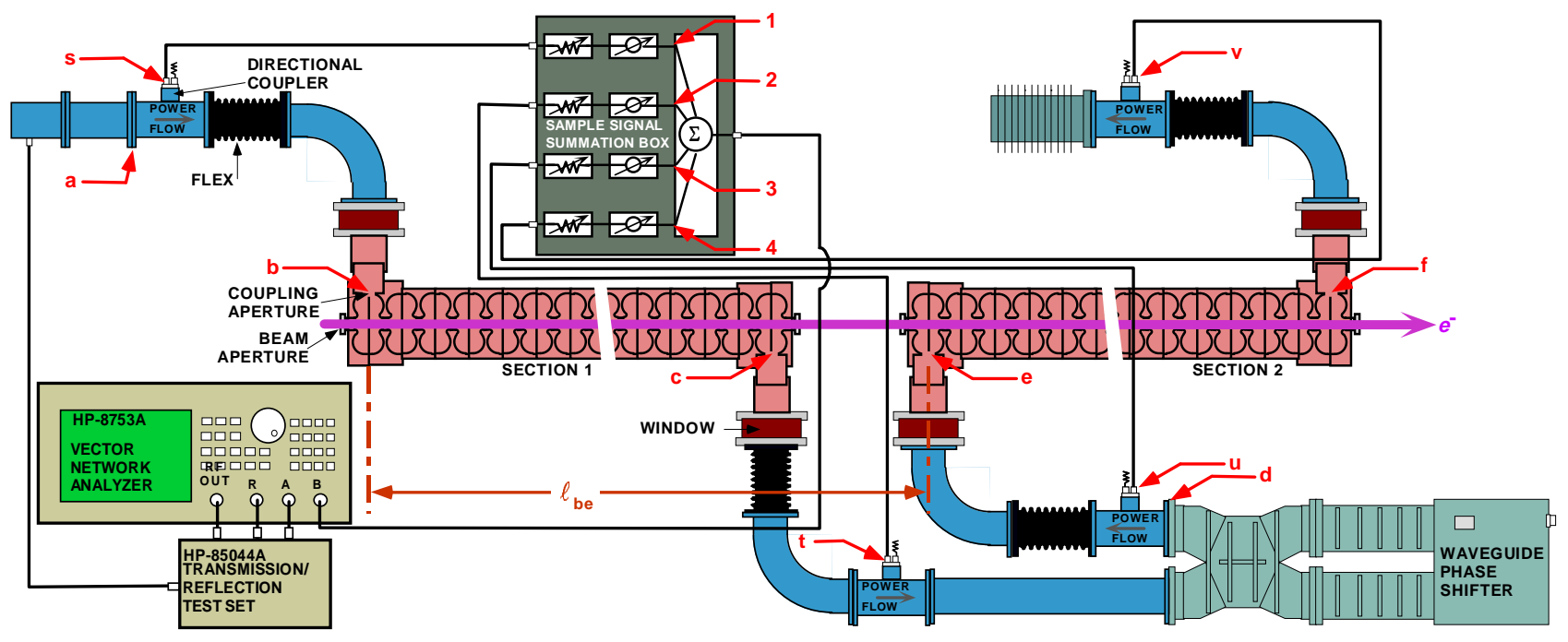

Figure 1: Representative configuration of the chief RF system components and network analyzer for measuring relative sample signals and adjusting summation box attenuators and phase shifters. Points of reference in the text are labeled with red arrows. Network analyzer is connected to the linac network in place of the klystron at point a.

\section{DETERMINING WEIGHTS}

To determine the best choice for the weighting factors $D_{b}, D_{c} \ldots$, a simple spread sheet model of the accelerating voltage in a typical traveling wave section of the proposed linac has been developed. Vector accelerating fields are computed for each cell, given the power entering from the upstream cell, local shunt impedance, and group velocity, after subtracting power transferred to the beam and applying a specified cell phase advance error $\phi_{e r r}$. The vector addition of the 18 cell voltage phasors of a second generation section described previously [1]

$$
V_{\text {true }}=\sum_{i=1}^{18} V_{i}
$$

is shown in Fig. 2 (solid lines) for five choices of phase advance error. For comparison, two linear combinations of the first and last cell voltage phasors are also shown: (1) the "unweighted" average of the first and last cell voltages multiplied by the number of cells; i.e.

$$
V_{\text {unwt }}=18\left(\frac{V_{l}+V_{18}}{2}\right)=9\left(V_{l}+V_{l 8}\right),
$$

shown as dotted lines, and (2) the weighted average

$$
V_{w t}=D_{1} V_{1}+D_{18} V_{18},
$$

shown as dashed lines, where weights $D_{l}=8.26$ and $D_{l 8}$ $=9.86$ are chosen so that

$$
\lim _{\phi_{e r t} \rightarrow 0} V_{w t}=\lim _{\phi_{c r t} \rightarrow 0} V_{\text {true }} .
$$

The first linear combination, while an improvement over the historical approach (which essentially delivers a result extending along the real axis to the red cross at
15.8 units regardless of $\phi_{\text {err }}$ ) tracks the true section voltage rather imperfectly, giving a net phase error even at very low $\phi_{e r r}$ and giving a significant amplitude error at larger $\phi_{e r r}$.

The second linear combination's weights are chosen so that it gives the correct value at a (low) $\phi_{e r r}$ of $0.1 \%$ cell. The dashed lines in Fig. 2 show that this approach corrects the phase error fairly well even for higher $\phi_{e r r}$, although the amplitude error remains for larger values of phase advance error.

The next question is how well this linear combination performs when there is beam loading. For the case $\phi_{e r r}=0$ (i.e., phase advance per cell is exactly the nominal $135^{\circ}$ ), the effect of beam loading has been modeled with an input power of $10 \mathrm{MW}$ for beam currents from zero to

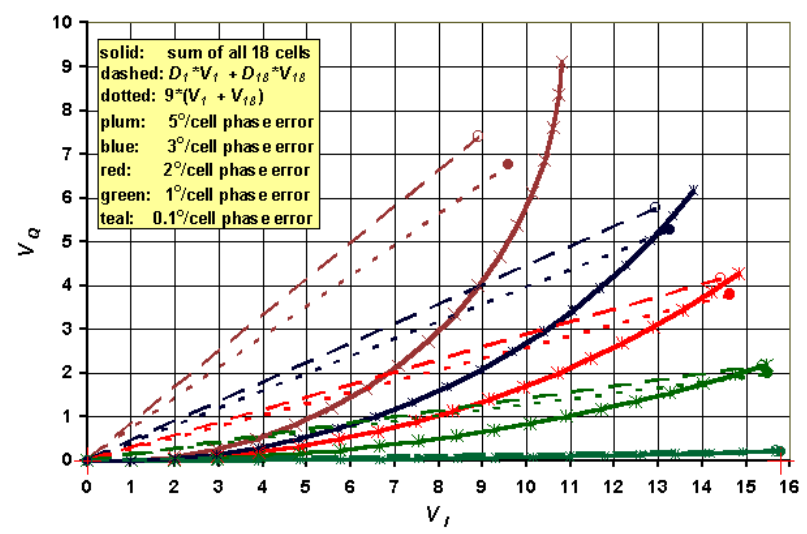

Figure 2: $V_{\text {true }}$ (solid lines), $V_{\text {unwt }}$ (dotted lines), and $V_{w t}$ (dashed lines) in the 18-cell constant impedance linac section, with phase advance error as a parameter. 
Table 1. FEL linac section parameters impacting relative sample signal strength. Total section attenuation $A_{s e c}$ is measured. Values of $v_{g} / c$ and $R_{s} / Q_{o}$ are computed [2]. Values of $D$ are determined using spread sheet model described above. Note: L band RF systems of this linac are numbered 3 through 6.

\begin{tabular}{|c|c|c|c|l|c|c|c|c|c|}
\hline $\begin{array}{c}\text { Section Type } \\
\text { Designation }\end{array}$ & Location(s)* & $\begin{array}{c}\text { No. } \\
\text { cells }\end{array}$ & $\begin{array}{c}\boldsymbol{A}_{\text {sec }} \\
(\mathbf{d B})\end{array}$ & End & $\begin{array}{c}\text { bore } \\
(\mathbf{i n})\end{array}$ & $\boldsymbol{v}_{\boldsymbol{g}} / \boldsymbol{c}$ & $\begin{array}{c}\boldsymbol{R}_{s} / \boldsymbol{Q}_{o} \\
(\Omega)\end{array}$ & $\begin{array}{c}\boldsymbol{R}_{\text {cell }} \\
(\mathbf{M} \Omega)\end{array}$ & $\boldsymbol{D}$ \\
\hline HAP/ & 4U, 4D, & 18 & 2.6 & IN & 2.2 & .0036 & 84.5 & .1106 & 8.24 \\
\cline { 5 - 10 } MOPA & 5D, 6U, 6D & & & OUT & 2.2 & .0036 & 84.5 & .1106 & 9.88 \\
\hline $\begin{array}{c}\text { HAP } \\
\text { Prototype }\end{array}$ & 3D & 25 & 2.2 & IN & 2.92 & .0167 & 62.8 & .0177 & 21.10 \\
\cline { 5 - 10 } & & & OUT & 2.18 & .0035 & 84.7 & .1140 & 7.63 \\
\hline $\begin{array}{c}\text { Original FEL } \\
\text { Oscillator }\end{array}$ & $5 \mathrm{U}$ & 35 & 4.3 & IN & 2.51 & .0074 & 75.2 & .0479 & 22.42 \\
\cline { 5 - 9 } & & & & OUT & 2.07 & .0026 & 87.7 & .1590 & 14.49 \\
\hline
\end{tabular}

*Numeral indicates RF system. A/B designates upstream/downstream in beam line order.

$0.5 \mathrm{~A}$. Even at beam currents up to 5 times nominal for the upgraded linac, resulting in a $24 \%$ droop in accelerating voltage, the relative amplitude error in the weighted average never exceeds $1.4 \%$, and is less than $0.25 \%$ at the $0.1-\mathrm{A}$ nominal beam current, within the acceptable range.

The numerical values found for the $D$ 's are specific to the section modeled; i.e., the values must be recomputed if structure parameters such as number of cells and impedance profile change. Results for the three section types used in the new linac are listed in Table 1.

\section{CONDITIONS ON SAMPLE SIGNALS}

Eq. 1 being established as the expression of the physical quantity which we seek to control, with $D$ coefficients in that equation determined as described above, it remains to write down the phase and amplitude relations which must be maintained between the sample signals presented to the power combiner in order for the combined signal to be proportional to $\boldsymbol{V}_{\text {ssstem }}$. The phase and level of each siample signal are measured in turn with the other sample lines disconnected, using the test configuration shown in Fig. 1. It can be shown [3] that, provided the linac sections and waveguide phase shifter are correctly tuned to preserve beam synchronicity with the $\mathrm{RF}$ wave, all the sample signal paths must have the same phase length from point $a$ in Fig. 1 to the summing junction.

It can further be shown that the sample signal strengths must be related by

$$
\begin{aligned}
& \frac{P_{2}}{P_{I}}=A_{\text {sec }} \frac{R_{c}}{R_{b}}\left(\frac{D_{c}}{D_{b}}\right)^{2}, \\
& \frac{P_{3}}{P_{I}}=A_{\text {sec }} \frac{R_{e}}{R_{b}}\left(\frac{D_{e}}{D_{b}}\right)^{2}, \text { and } \\
& \frac{P_{4}}{P_{I}}=A_{\text {sec }} A_{s e c 2} \frac{R_{f}}{R_{b}}\left(\frac{D_{f}}{D_{b}}\right)^{2},
\end{aligned}
$$

where $A_{\text {sec }}$ and $A_{\text {sec } 2}$ are the power attenuation in the first and second sections respectively, and $R_{b, . f}=\frac{2 \varphi}{v_{g} / c}\left(\frac{R_{s}}{Q_{o}}\right)$, where $\varphi=3 \pi / 4$ and where $\left(R_{s} / Q_{o}\right)$ and $\left(v_{s} / c\right)$ are the local section parameters at the appropriate end cells (Table 1).

Using the linac section parameter values shown in Table 1 in Eqs. 2-4, the relative levels can be found for each of the four L band RF systems in the upgraded linac. The results each system appear in Table 2. Note that System 3 has only one section which operates as an accelerator section, and that in System 5 the sections appear in reverse order on the beam line so that Section $5 \mathrm{D}$ is the first section in the RF circuit.

Table 2: Relative levels of sample signals required for summed output to be proportional to system accelerating voltage.

\begin{tabular}{|r|c|c|c|c|}
\hline RF System & 3 & 4 & 5 & 6 \\
\hline \hline$P_{2} / P_{I}(\mathrm{~dB})$ & -1.9 & -1.1 & -1.1 & -1.1 \\
\hline$P_{3} / P_{\perp}(\mathrm{dB})$ & & -2.6 & +2.4 & -2.6 \\
\hline$P_{4} / P_{\perp}(\mathrm{dB})$ & & -3.7 & -0.4 & -3.7 \\
\hline
\end{tabular}

\section{REFERENCES}

[1] A.M. Vetter, et al., "First Operation of the HAP/MOPA Traveling Wave Structure at Boeing FEL”, Proc. 1997 Particle Accelerator Conference, Vancouver BC, May 12-16, 1997, p. 2950 (1998).

[2] A.M. Vetter, "Performance Expectations for Original HAP Linac Sections", Boeing Free Electron Laser Program report FEL/AT97-001, March, 1997.

[3] A.M. Vetter, "Optimally Weighted Sample Signal Combination for Traveling Wave Linac Section Pairs", Boeing Free Electron Laser Program report FEL/AT99-003, March, 1999. 